\title{
L'utilizzo del gel piastrinico (prp) nella chirurgia di revisione della componente acetabolare d'anca
}

G.Sessa, L. Costarella, F.R. Evola

Clinica Ortopedica Università degli Studi di Catania

ABSTRACT The use of platelet gel (PRP) in revision surgery of the acetabular component of the hip

The aseptic loosening of the acetabular cup is a frequently complication in THA.

The most important problem in the revision surgery is the bone loss that complicates the surgical procedures and thus the success of the implant in the long term.

The homologous and heterologous bone grafts often represent the most widely used solution either in the morselized form, as a filler,or as structural graft with mechanical function.

These tissues, however, have biological limitations and lose most of osteogenic and osteoinductive capacity; all this leads to the use of growth factors such autologous platelet rich plasma(P.R.P.). At the Institute of Orthopaedic and Traumatology Clinic of Catania between September 2004 and December 2012 were undergoing surgery for acetabular hip revision 26 patients;in 18 cases the PRP has been associated with homologous bone, while in 6 cases with equine bone.

At a mean follow-up of 5 years (range 9-4 ys) the clinical and radiographic results were good in 23 cases, while, in 3 cases there has been an unsatisfactory result. We have not proved significant differences between the two groups of patients treated with homologous or equine bone.

To further assess the real effectiveness will have to be carried out randomized studies on a large scale and precisely determine protocols for application and provide clear guidelines.

\section{Introduzione}

La mobilizzazione asettica delle componenti protesiche dell'anca e soprattutto del cotile, rappresenta una complicanza frequente nella chirurgia protesica dell'anca. La problematica principale nella successiva chirurgia di revisione è rappresentata dalla perdita di tessuto osseo (bone loss) dell'ospite; la gravità della perdita ossea e la sua riparazione condizionano la strategia chirurgica e di conseguenza il successo dell'impianto a lungo termine [1]

Per far fronte a tale carenza, gli innesti omologhi e/o eterologhi rappresentano spesso la soluzione più utilizzata ed in particolare nella ricostruzione acetabolare ove il oro impiego trova indicazione più spesso in forma morcellizzata a scopo riempitivo, ma anche come innesti strutturati con finalità di supporto meccanico.

L'osso omologo e/o eterologo ha tuttavia delle limitazioni biologiche legate alla sua processazione, se infatti mantiene inalterate le sue proprietà di osteoconduzione,perde di fatto rispetto all'osso autologo gran parte della capacità osteoinduttiva e osteogenetica [2]; tutto ciò spinge maggiormente ad utilizzare dei fattori di crescita tali da ovviare a queste carenze osteogenetiche. Limpiego del gel piastrinico (PRP) rappresenta una metodica disponibile per la rigenerazione di tessuto osseo in sedi deficitarie.

E' noto che le piastrine, oltre a svolgere un ruolo fondamentale nell'emostasi, contengono numerosi fattori di crescita coinvolti nei meccanismi di generazione di tessuto osseo [3].

Un composto ad elevata concentrazione piastrinica è pertanto, in linea teorica, in grado di stimolare ed accelerare la rigenerazione Limpiego del plasma arricchito di piastrine potrebbe pertanto migliorare e sveltire i meccanismi di attecchimento degli innesti ossei autologhi utilizzati per la correzione dei difetti.

Presso l'Istituto di Clinica Ortopedica e Traumatologica di Catania, nell'arco temporale compreso tra Settembre 2004 e Dicembre 2012 , sono stati sottoposti ad intervento chirurgico di revisione acetabolare dell'anca 26 pazienti: in 18 casi le PRP sono state associate con osso omologo, mentre in 6 casi sono state associate ad osso equino.

Di questi 10 erano donne e 16 uomini con età media di 68 anni (range: 62-78 aa.)

Secondo la classificazione G.I.R. 4 pz. rientravano nel tipo I, 14 nel tipo II e 8 nel tipo III

Tutti sono stati valutati da un punto di vista clinico e radiografico a 1-3-6-12 mesi dall'intervento e quindi ad intervalli annuali.

Il follow-up medio è di 5 anni (range 9-4 aa.)

Al momento dell'intervento nessun paziente presentava segni clinici e valori degli esami ematochimici suggestivi per un processo infettivo.

Non si sono registrate complicanze nel post-operatorio. Nella valutazione dei risultati a distanza sono stati registrati risultati clinici soddisfacenti in 23 casi, con assenza di sintomatologia algica, buona articolarità e deambula zione libera; solo in 3 casi, in pz. affetti da A.R., si è avuto un risultato parzialmente soddisfacente con lieve dolore durante le normali attività quotidiane e con deambulazione che avveniva con uso di bastone canadesi, di questi in 2 pazienti era stato utilizzato osso equino

Da un punto di vista radiologico guidata di tessuto osseo [4]. si è assistito ad un precoce e buon processo di osteointegrazione (in media 6 mesi) con ristrutturazione dei difetti ossei e rimodella mento dei trapianti, confermando così il presupposto teorico della capacità ricostruttiva, in associazione ad innesti ossei, del gel piastrinico. Il razionale alla base dell'impiego del gel piastrinico sta nella constatazione che le piastrine contengono diversi fattori $\mathrm{d}$ crescita coinvolti nella regolazione dei processi di generazione e di guarigione tessutale [5].

Leterotrapianto, invece, svolge funzioni di carrier e rappresent una sorgente supplementare di cellule staminali, oltre a permettere di colmare le perdite di sostanza ossea. Stimolazione biologica e ricostruzione parietale-meccanica pertanto sono indispensabili nelle revisioni con difetti ossei. Lintervento di reimpianto di protesi d'anca comporta sempre scelte difficili, per ricreare i parametri di una biomeccanica alterata dal fallimento di procedure chirurgiche precedenti, dalla selezione dell'impianto per forma e materiali alla definizione di qualità e quantità di osso o sostituti
D01 10.1007/510261-013-0053-2

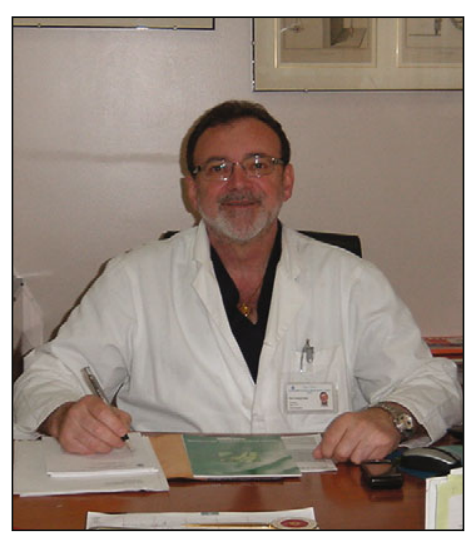

G. Sessa

I.O.R.S. Varese- Settembre

2. Civinini R-Capone A-D'Arienzo M.-Innocenti M.-Gusso M.I.( 2004) Gli innesti ossei ed i sostituti dell'osso nelle revisioni acetabolari. G.I.O.T. 30(suppl.1): 94-98

3. Zimmermann $\mathrm{R}$ et al (2001) Different preparation methods to obtain platelet components as a source of growth factors for local application. Trasfusion 41(10):1217-1224

4. Canalis E.-McCarthy TL-Centrella M (1989) Effects of platelet-derived growth factor on bone formation in vitro. J Cel. Physiol 140:530-537

5. Marx RE, Carlson ER, Eichstaed RM et al (1998) Platelet-rich plasma: Growth factor enhancement for bone grafts. Oral Surg Oral Med Oral Pathol 85. 638Orat

\section{Bibliografia}

1. Sessa G.-Costarella L.-Spicuzza A.-Pavone V (2005) Prime esperienze con l'utilizzo di gel piastrinico nella chirurgia di revisione della componente acetabolare d'anca. Abstract VII Congresso

. Castagna A, Randelli PS, Garofalo R (2010) Biologia della guarigione della riparazione della cuffia dei rotatori e ruolo dei fatton di crescita. G.I.O.T.36(suppl.1) 258-260 